\title{
Smart bioelectronics and biomedical devices
}

\author{
Nanjia Zhou ${ }^{1,2}$ (D) Liang $\mathrm{Ma}^{3,4}$ \\ Received: 17 November 2021 / Accepted: 22 November 2021 / Published online: 14 January 2022 \\ (c) Zhejiang University Press 2022
}

The development of smart bioelectronics and biomedical devices has significantly advanced the field of biomedical engineering, enabling a myriad of applications from basic biomedical research to clinical medicine and implants. Standing at the intersection of materials science, mechanical, electrical, and bioengineering, this fascinating field has attracted considerable attention from researchers all over the world, providing new healthcare solutions which were impossible before. From brain-machine interface to biomedical nano/micro-robots, different materials and manufacturing methodologies have been developed to advance the functionalities of the bioelectronic systems to meet the ever-increasing demand of biological and bioinspired applications (Fig. 1).

\section{Materials for bioelectronics and biomedical devices}

From materials point of view, bioelectronics and biomedical devices are mostly composed of conductive, dielectric, and semiconductor materials, as well as soft substrates/encapsulations. These systems which can interface with human bodies via electrical stimulation and recording of biological signals are of great interest in emerging neural science and engineering [1], diagnostics [2], therapy [3], and wearable and implantable devices [4]. The fab-

$凶$ Nanjia Zhou

zhounanjia@westlake.edu.cn

$凶$ Liang Ma

liangma@zju.edu.cn

1 Key Laboratory of 3D Micro/Nano Fabrication and Characterization of Zhejiang Province, School of Engineering, Westlake University, Hangzhou 310024, China

2 Institute of Advanced Technology, Westlake Institute for Advanced Study, Hangzhou 310024, China

3 State Key Laboratory of Fluid Power and Mechatronic Systems, Zhejiang University, Hangzhou 310058, China

4 School of Mechanical Engineering, Zhejiang University, Hangzhou 310058, China rication of bioelectronics combines the careful design of deformable circuits, integration of rigid and planar semiconductor devices, and the engineering of soft and stretchable encapsulations to realize a better mechanical matching with soft biological tissues and even growing organisms, enabling their constant communication with human body.

The biocompatible metals such as gold were engineered into serpentine shapes to enabling its stretchability as conducting circuit materials, by further integration of these engineered stretchable circuits with semiconductor into a thin and flexible polydimethylsiloxane (PDMS)based encapsulations via "soft lithographic" technologies [5]; several types of bioelectronics were fabricated including epidermal tactile/chemo-sensors [6], implantable cardiac pacemaker [7], optoelectronics [8], etc., which demonstrated their applications in biomedical research and potential clinical treatments. The employment of degradable metal and metal oxides as conducting and dielectric materials, respectively, and bioresorbable silk or poly(lacticco-glycolic acid) (PLGA), transient bioelectronics can be manufactured [9-12]. However, there still could be challenges with formation of scar tissue between bioelectronics and biological tissues during long-term implantation, resulting in the increase in interfacial impedance [13].

Hydrogels have emerged as a promising candidate for fabrication of next-generation bioelectronics, due to their similarity to biological tissues, water infiltrability and versatility in electrical, mechanical, and biofunctional engineering. Either synthetic polymers like polyvinyl alcohol (PVA), polyacrylic acid (PAA), polyacrylamide (PAAm), poly(2-hydroxyethyl methacrylate) (PHEMA), etc., or natural macromolecules such as polysaccharides, gelatin and proteins, etc., can be utilized to synthesized biocompatible hydrogels. By further dissolving electrolyte molecules such as $\mathrm{LiCl}, \mathrm{NaCl}, \mathrm{KCl}$ or even zwitterionic molecules into hydrogel matrix, ionic conductive can be achieved [14, 15]. Theses ionic conductive hydrogels based electronics were also named as ionotronics, which have found their applications in epidermal sensors, artificial axons and soft 
Fig. 1 Materials, manufacturing and system validation in bioelectronic devices and systems

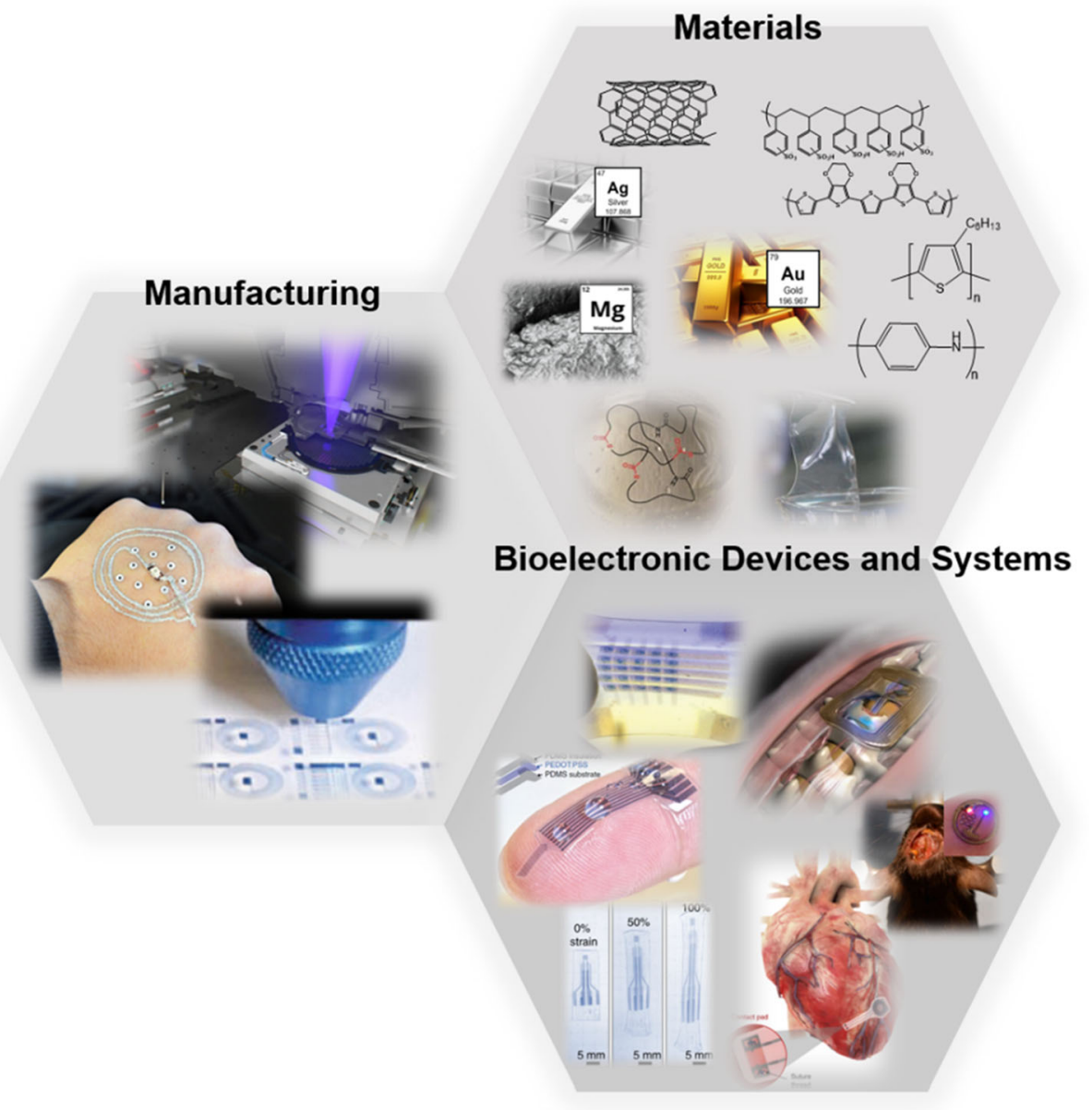

actuators [16]. However, their low conductivity and ionic conductive nature hindered their applications in in vivo tests.

Another important category of materials in the fabrication of bioelectronics is the amphiphilic conducting polymers, such as poly(3,4-ethylenedioxythiophene) polystyrene sulfonate (PEDOT/PSS), polypyrrole (PPy), poly(3-hexylthiophene-2,5-diyl) (P3HT) and polyaniline (PANI). Their high electric conductivity compared with ionically conductive hydrogels makes them promising candidates in fabrication of implantable bioelectronics, such as neural probes that can monitor the electrophysiological signals in mouse brain [17], implantable and morphing electronics for long-term chronic electrical stimulation and monitoring [18], cellular scaffolds [19] and so on.

Recently, composite conductive hydrogels, synthesized via the incorporation of conductive fillers into hydrogel matrix, have emerged as another alternative candidate for bioelectronics. Harnessing the high electrical conductivity from conductive materials like PEDOT/PSS, carbon nanotubes, graphene etc., as well as the softness and stretchability from hydrogels, the composite materials could exhibit both high electrical conductivity up to about
$50 \mathrm{~S} / \mathrm{m}$ and mechanical stretchability up to about $600 \%$ [20].

\section{New manufacturing technologies for bioelectronics and biomedical device}

From a manufacturing and structural design point of view, conventional manufacturing methods rely on nanofabrication methods such as photolithography, e-beam evaporation, and sputtering of conductive, dielectric, and semiconductor features on flexible substrates [21]. Rogers group also invented a series of transfer printing processes which are compatible with silicon electronics fabrication featuring complex logic functionalities. Over the years, a series of transfer printed systems have been demonstrated, such as cerebral cortex mapping [10], cardiac electrophysiology [22], to name a few. To overcome the limitations of planar device geometries which are often incompatible with biological systems, recent bioelectronics employ conformal additive stamp (CAS) printing technology to transfer complex silicon electronics onto non-flat surfaces [23]. Another strategy of significant interest is the employment of mechanically guided 
buckling $[24,25]$ which enables scalable, high-resolution fabrication of 3D structures. To conform with biological tissues or blood vessels [26], flexible bioelectronics can also be wrapped around arteries of various sizes to allow the accurate measurement of blood flow.

As an alternative to conventional nanofabrication methods, additive manufacturing methods have recently emerged due to their advantages such as low-cost, rapid prototyping, and customization. They also allow the patterning of many materials which are otherwise difficult to integrate using conventional methods. Among different additive approaches, screen and inkjet printing have been the most reported methods due to their easy access and relatively long history [27]. Most reports focus on maskless patterning of low concentration dielectric and conductive inks [28]. Harnessing a diverse range of functional materials, direct ink writing (DIW) has recently emerged as a versatile platform for bioelectronics and biosystem fabrication. Yuk et al. [17] introduced 3D printed soft neural probes for electrophysiological recording in the mouse dorsal hippocampus (dHPC) using PEDOT/PSS ink. For multimaterial DIW, Lind et al. [29] demonstrated an example of instrumented cardiac microphysiological devices for drug response study using a variety of sacrificial, elastomer, conductive and dielectric materials.

Focusing on new materials, design, and manufacturing aspects, we have put up together an exciting special issue on the topic of "Smart Bioelectronics and Biomedical Devices," emphasizing the bio-design and manufacturing aspects involved in bioelectronics and biomedical devices, with new materials, design, fabrication, and application aspects. We begin with an editorial by Han et al. [30] discussing the additive manufacturing of nerve conduits. The editorial outlines the advantages of additive manufacturing in mimicking the topological shape, biocompatibility and mechanical properties required for nerve tissue extracellular matrix (ECM), and progresses and challenges with printable biomaterials and their high-resolution processing.

One perspective by Huang et al. covers the topic of minimally invasive biosensor for glucose monitoring especially using microneedle technology with the aim to safely and accurately detect subcutaneous microenvironment changes associated with diabetes complications.

A collection of research articles then ensues. Huang et al. [31] developed a stretchable self-powered biosensor integrating enzymatic biofuel cells which serve as self-powered sensing modules. The lactate and glucose enzymatic biofuel cells (EBFCs) sensors were fabricated by a series of spray coating and casting method, together with a lithography-enabled microfluidic device for efficient sweat collection and sensing. Jing et al. [32] developed a new conductive and transparent dipeptide hydrogel ink using hydrophilic conductive polydopamine (PDA)-doped polypyrrole (PPy) nanoparticle incorporated dipeptide hydrogel. This strategy allows good conductivity and transparency of the material and enables applications for body-adhered signal detection. Li et al. [33] applied femtosecond laser processing to modify Pt-Ir neural electrode with superwicking behavior, which offers highly improved electrical performance and wettability. Thanks to the hierarchical surface micro/nanostructures enabled by laser processing, the electrode shows the increased maximum charge storage capacity which makes them promising for use in clinical neural electrodes. Wang et al. [34] designed a class of pneumatic muscle-like actuators, termed highly mimetic skeletal muscle (HimiSK), consisting of parallelly aligned contractile units in a flexible matrix. The contractile units and flexible matrix mimic muscle fiber and connective tissue, respectively, enabling 3D actuation upon activation and showing intrinsic force-velocity and force-length behaviors similar to biological muscles. Zeng et al. [35] developed a bio-inspired 4D printing layout strategy by designing the area delineation and shape memory properties of shape memory polymers (SMPs). Zhang et al. [36] developed a novel pneumatically assisted atomization device (PAAD) which allows uniform living cell delivery with low pressure. The sprayed cells maintained high viability and differentiation, making them ideal for clinical cell therapy and wound healing. Zhang et al. developed a 3D printed microfluidic gradient concentration chip using digital light processing (DLP) for rapid antibiotic susceptibility testing. The obtained gradient concentration environment of antibiotics provides a rapid testing method for measuring bacterial susceptibility to antibiotics in clinical settings. Hesselmann et al. [37] proposed a novel design for $3 \mathrm{D}$ potting of membrane modules based on a multiaxial centrifugation process, which could improve flow path and lower stagnation volume, and ultimately improve efficiency for artificial lungs. Lastly, amid Covid-19 pandemic, Park et al. [38] designed and tested cost-effective, rapidly scalable ventilator (ALIVE Vent) using commercially available components. This solution might offer life-saving solutions especially for people in resource-scarce regions.

The special issue also collects five review articles across several important areas of bio-design and manufacturing. Santoni et al. provided an extensive outlook on scientific literature and patents on the topics of bioprinting, showing that the field is undergoing rapid expansion. Echoing the research article by Huang et al. [31] in this issue, Heng et al. provided a review on wearable flexible sensors for sweat analysis. The recent research focuses on sweat sensors with a diverse form factors and materials. The review also covers different mechanisms of sensing as well as their advantages and challenges. Lim et al. [39] discussed the requirement of bioreactor design for tissue engineering, which should provide a biomimetic physiological platform by optimizing material design, mass transfer, mechanical and electrical stimulation. Lin et al. [40] summarized the exciting topic of biorobots which have the advantages of energy efficiency and miniaturization. This 
review covers the progress of biohybrid robots regarding power sources, supporting biomaterials and structures developed in the recent years. Finally, the work of Willemen et al. [41] covers various 3D and 4D printing techniques and their applications in the pharmaceutical field as drug delivery systems and personalized medicine. It is hoped that this special issue will serve as a collection of research and views from different angles and bring new insights to the growing field of bioelectronic devices and systems, leading to new inspiration to the field and expanding to possibilities of smart bioelectronics and biomedical devices for many practical and clinical applications.

\section{References}

1. Sunwoo SH, Han SI, Joo H et al (2020) Advances in soft bioelectronics for brain research and clinical neuroengineering. Matter 3(6):1923-1947. https://doi.org/10.1016/j.matt.2020.10.020

2. Lee SH, Kim YS, Yeo WH (2021) Advances in microsensors and wearable bioelectronics for digital stethoscopes in health monitoring and disease diagnosis. Adv Healthc Mater 10(22):2101400. https://doi.org/10.1002/adhm.202101400

3. Wu H, Gao W, Yin Z (2017) Materials, devices and systems of soft bioelectronics for precision therapy. Adv Healthc Mater 6(10):1700017. https://doi.org/10.1002/adhm.201700017

4. Sunwoo SH, Ha KH, Lee S et al (2021) Wearable and implantable soft bioelectronics: device designs and material strategies. Ann Rev Chem Biomol Eng 12(1):359-391. https://doi.org/10.1146/ annurev-chembioeng-101420-024336

5. Kim DH, Lu N, Ma R et al (2011) Epidermal electronics. Science 333(6044):838-843. https://doi.org/10.1126/science.1206157

6. Ghaffari R, Rogers JA, Ray TR (2021) Recent progress, challenges, and opportunities for wearable biochemical sensors for sweat analysis. Sens Actuat B Chem 332:129447. https://doi.org/10.1016/j. snb.2021.129447

7. Gutruf P, Yin RT, Lee KB et al (2019) Wireless, battery-free, fully implantable multimodal and multisite pacemakers for applications in small animal models. Nat Commun 10(1):5742. https://doi.org/ 10.1038/s41467-019-13637-w

8. Kim TI, McCall JG, Jung YH et al (2013) Injectable, cellularscale optoelectronics with applications for wireless optogenetics. Science 340(6129):211-216. https://doi.org/10.1126/science. 1232437

9. Brenckle MA, Cheng H, Hwang S et al (2015) Modulated degradation of transient electronic devices through multilayer silk fibroin pockets. ACS Appl Mater Interf 7(36):19870-19875. https://doi. org/10.1021/acsami.5b06059

10. Yu KJ, Kuzum D, Hwang SW et al (2016) Bioresorbable silicon electronics for transient spatiotemporal mapping of electrical activity from the cerebral cortex. Nat Mater 15(7):782-791. https://doi. org/10.1038/nmat4624

11. Choi YS, Koo J, Lee YJ et al (2020) Biodegradable polyanhydrides as encapsulation layers for transient electronics. Adv Funct Mater 30(31):2000941. https://doi.org/10.1002/adfm.202000941

12. Choi YS, Yin RT, Pfenniger A et al (2021) Fully implantable and bioresorbable cardiac pacemakers without leads or batteries. Nat Biotechnol 39(10):1228-1238. https://doi.org/10.1038/ s41587-021-00948-x

13. Yuk H, Lu B, Zhao X (2019) Hydrogel bioelectronics. Chem Soc Rev 48(6):1642-1667. https://doi.org/10.1039/C8CS00595H
14. Keplinger C, Sun JY, Foo CC et al (2013) Stretchable, transparent, ionic conductors. Science 341(6149):984-987. https://doi.org/10. $1126 /$ science. 1240228

15. Zhang W, Wu B, Sun S et al (2021) Skin-like mechanoresponsive self-healing ionic elastomer from supramolecular zwitterionic network. Nat Commun 12(1):4082. https://doi.org/10.1038/s41467021-24382-4

16. Yang C, Suo Z (2018) Hydrogel ionotronics. Nat Rev Mater 3(6): 125-142. https://doi.org/10.1038/s41578-018-0018-7

17. Yuk H, Lu B, Lin S et al (2020) 3D printing of conducting polymers. Nat Commun 11(1):1604. https://doi.org/10.1038/s41467020-15316-7

18. Liu Y, Li J, Song S et al (2020) Morphing electronics enable neuromodulation in growing tissue. Nat Biotechnol 38(9):1031-1036. https://doi.org/10.1038/s41587-020-0495-2

19. Feig VR, Santhanam S, McConnell KW et al (2021) Conducting polymer-based granular hydrogels for injectable 3D cell scaffolds. Adv Mater Technol 6(6):2100162. https://doi.org/10.1002/admt. 202100162

20. Wei H, Lei M, Zhang P et al (2021) Orthogonal photochemistryassisted printing of 3D tough and stretchable conductive hydrogels. Nat Commun 12(1):2082. https://doi.org/10.1038/s41467021-21869-y

21. Hwang SW, Tao H, Kim DH et al (2012) A physically transient form of silicon electronics. Science 337(6102):1640-1644. https:// doi.org/10.1126/science.1226325

22. Fang H, Yu KJ, Gloschat $\mathrm{C}$ et al (2017) Capacitively coupled arrays of multiplexed flexible silicon transistors for long-term cardiac electrophysiology. Nat Biomed Eng 1(3):0038. https://doi.org/10. 1038/s41551-017-0038

23. Sim K, Chen S, Li Z et al (2019) Three-dimensional curvy electronics created using conformal additive stamp printing. Nat Electron 2(10):471-479. https://doi.org/10.1038/s41928-019-0304-4

24. Luan H, Zhang Q, Liu TL et al (2021) Complex 3D microfluidic architectures formed by mechanically guided compressive buckling. Sci Adv 7(43):eabj3686. https://doi.org/10.1126/sciadv. abj3686

25. Zhao H, Li K, Han Met al (2019) Buckling and twisting of advanced materials into morphable 3D mesostructures. Proc Nat Acad Sci 116(27):13239. https://doi.org/10.1073/pnas.1901193116

26. Boutry CM, Beker L, Kaizawa Y et al (2019) Biodegradable and flexible arterial-pulse sensor for the wireless monitoring of blood flow. Nat Biomed Eng 3(1):47-57. https://doi.org/10.1038/s41551018-0336-5

27. Huang X, Liu Y, Hwang SW et al (2014) Biodegradable materials for multilayer transient printed circuit boards. Adv Mater 26(43):7371-7377. https://doi.org/10.1002/adma.201403164

28. Feng S, Tian Z, Wang J et al (2019) Laser sintering of Zn microparticles and its application in printable biodegradable electronics. Adv Electron Mater 5(3):1800693. https://doi.org/10.1002/aelm. 201800693

29. Lind JU, Busbee TA, Valentine $A D$ et al (2017) Instrumented cardiac microphysiological devices via multimaterial three-dimensional printing. Nat Mater 16(3):303-308. https://doi. org/10.1038/nmat4782

30. Han Y, Yin J (2021) Industry news: the additive manufacturing of nerve conduits for the treatment of peripheral nerve injury. Bio-Des Manuf. https://doi.org/10.1007/s42242-021-00166-z

31. Huang XC, Li JY, Liu YM et al (2021) Epidermal self-powered sweat sensors for glucose and lactate monitoring. Bio-Des Manuf. https://doi.org/10.1007/s42242-021-00156-1

32. Jing YF, Wang AH, Li JL et al (2021) Preparation of conductive and transparent dipeptide hydrogels for wearable biosensor. BioDes Manuf. https://doi.org/10.1007/s42242-021-00143-6

33. Li LZ, Jiang CQ, Li LM (2021) Hierarchical platinum-iridium neural electrodes structured by femtosecond laser for superwicking 
interface and superior charge storage capacity. Bio-Des Manuf. https://doi.org/10.1007/s42242-021-00160-5

34. Wang YJ, Liu CB, Ren LQ et al (2021) Bioinspired soft actuators with highly ordered skeletal muscle structures. Bio-Des Manuf. https://doi.org/10.1007/s42242-021-00148-1

35. Zeng SY, Feng YX, Gao YC et al (2021) Layout design and application of 4D-printing bio-inspired structures with programmable actuators. Bio-Des Manuf. https://doi.org/10.1007/s42242-02100146-3

36. Zhang LX, Yan XT, An L et al (2021) Novel pneumatically assisted atomization device for living cell delivery: application of sprayed mesenchymal stem cells for skin regeneration. Bio-Des Manuf. https://doi.org/10.1007/s42242-021-00144-5

37. Hesselmann F, Focke JM, Schlanstein PC et al (2021) Introducing 3D-potting: a novel production process for artificial membrane lungs with superior blood flow design. Bio-Des Manuf. https://doi. org/10.1007/s42242-021-00139-2

38. Park MH, Zhu YJ, Wang HJ et al (2021) From hardware store to hospital: a COVID-19-inspired, cost-effective, open-source, in vivo-validated ventilator for use in resource-scarce regions. BioDes Manuf. https://doi.org/10.1007/s42242-021-00164-1

39. Lim D, Renteria ES, Sime DS et al (2021) Bioreactor design and validation for manufacturing strategies in tissue engineering. BioDes Manuf. https://doi.org/10.1007/s42242-021-00154-3

40. Lin ZN, Jiang T, Shang JZ (2021) The emerging technology of biohybrid micro-robots: a review. Bio-Des Manuf. https://doi.org/ 10.1007/s42242-021-00135-6

41. Willemen NGA, Morsink MAJ, Veerman D et al (2021) From oral formulations to drug-eluting implants: using $3 \mathrm{D}$ and $4 \mathrm{D}$ printing to develop drug delivery systems and personalized medicine. Bio-Des Manuf. https://doi.org/10.1007/s42242-021-00157-0

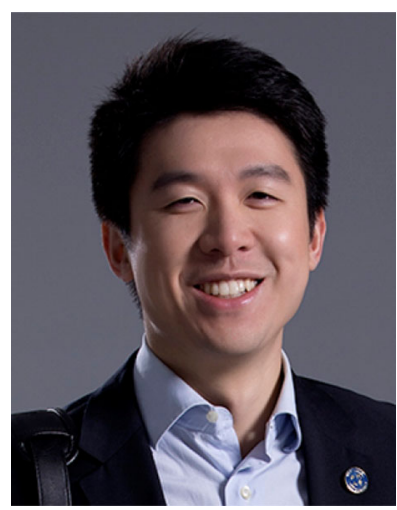

Guest Editor of the Special Issue: Dr. Nanjia Zhou received his Ph.D. from Northwestern University in Materials Science and Engineering in 2015 before moving to Harvard University as a Camille and Henry Dreyfus Postdoctoral Fellow. He started his academic career as an assistant professor in National University of Singapore in early 2018 before moving to Westlake University in September 2018. He is currently the PI of Intelligent Manufacturing Lab in School of Engineering. His current research focuses on developing novel functional materials for $3 \mathrm{D}$ printed soft electronics, optics, robotics, and bioelectronics, as well as new 3D printing processes which will enable high resolution, high throughput, digitally programmable multi-material assembly. He is also the founder of Enovate3D, which focuses on 1-10 micrometer feature size electronics 3D printing technologies for mass production. $\mathrm{He}$ serves as an Associate Editor of Bio-Design and Manufacturing since 2019.

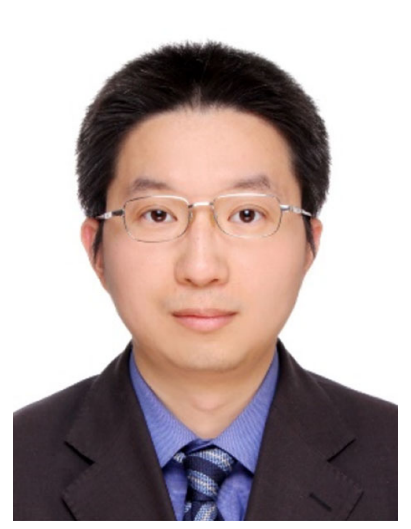

Guest Editor of the Special Issue: Dr. Liang Ma received both B.Eng. in Materials Science and Engineering and B.S. in Bioinformatics from Zhejiang University, China in 2005 and Ph.D. degree in Mechanical Engineering from University of Washington in 2012. $\mathrm{He}$ is now an Associate Professor in School of Mechanical Engineering, Zhejiang University. He has published more than 45 journal papers such as Small Methods, Biomaterials, Engineering and Biofabrication. His research interests include high resolution 3D bioprinter development and 3D bioprinting of tissues and organs especially tumor in vitro models and organs-on-chips. He severs as the Deputy Director of the editorial office of Bio-Design and Manufacturing. 